\title{
Usefulness of F-18 FDG PET/CT in subcutaneous panniculitis-like $T$ cell lymphoma: disease extent and treatment response evaluation
}

\author{
Jin-Suk Kim¹, Young Jin Jeong², Myung-Hee Sohn³,5, Hwan-Jeong Jeong ${ }^{3,5}$, Seok Tae Lim³, \\ Dong Wook Kim³,5, Jae-Yong Kwak ${ }^{4,5}$, Chang-Yeol Yim ${ }^{4,5}$ \\ ${ }^{1}$ Department of Nuclear Medicine, Konyang University Hospital, Daejeon, South Korea \\ 2 Department of Nuclear Medicine, Dong-A University Medical Center, Busan, South Korea \\ ${ }^{3}$ Department of Nuclear Medicine, ${ }^{4}$ Department of Internal Medicine and ${ }^{5}$ Research Institute of Clinical Medicine, \\ Chonbuk National University Medical School and Hospital, Jeonju, Jeonbuk, South Korea
}

Radiol Oncol 2012; 46(4): 279-283.

Received 30 December 2011

Accepted 21 January 2012

Correspondence to: Myung-Hee Sohn, M.D., Department of Nuclear Medicine, Chonbuk National University Medical School and Hospital, San 2-20 Geumam-dong, Deokjin-gu, Jeonju, Jeonbuk, 561-180, South Korea. Phone: +82-63-250-1174; Fax: +82-63-255-1172;

E-mail: mhsohn@jbnu.ac.kr

Disclosure: The authors have no conflicts of interest to disclose.

Background. Subcutaneous panniculitis-like T-cell lymphoma (SPTCL) is a rare form of cutaneous lymphomas, accounting for less than $1 \%$ of cases of non-Hodgkin's lymphoma. Fluorine-18 fluorodeoxyglucose (F-18 FDG) positronemission tomography/computed tomography (PET/CT) findings of SPTCL before and after treatment were rarely reported.

Case report. We report a case of SPTCL in which F-18 FDG PET/CT showed increased FDG accumulations in numerous subcutaneous nodules without extracutaneous disease. Contrast-enhanced CT during F-18 FDG PET/CT showed multiple minimally enhancing nodules with an infiltrative pattern in the subcutaneous layer throughout the body. Follow-up F-18 FDG PET/CT after three cycles of CHOP chemotherapy showed a complete metabolic remission of the lesions.

Conclusions. F-18 FDG PET/CT is suggested to be useful in assessing the disease activity, extent and treatment response in SPTCL.

Key words: subcutaneous panniculitis-like T-cell lymphoma; F-18 FDG; PET/CT

\section{Introduction}

Subcutaneous panniculitis-like T-cell lymphoma (SPTCL) is a rare disorder that is often confused with panniculitis. Patients usually present with multiple subcutaneous nodules on the extremities and trunk without visceral disease. Systemic symptoms such as fever, fatigue, and weigh loss may be present. The disease may be complicated by hemophagocytic syndrome, which is often associated with a rapidly progressive course. ${ }^{1}$ The diagnosis of SPTCL is currently based on clinical and histological findings. ${ }^{2} \mathrm{CT}$ is a noninvasive imaging modality that is widely used for staging in patients with lymphoma ${ }^{3}$, but it does not provide much information in cutaneous lesions. ${ }^{4}$

We report herein a patient with SPTCL in whom F-18 FDG PET/CT was valuable in assessing the extent of the disease and the treatment response.

\section{Case report}

A 30-year-old man presented with a 10-year history of multiple subcutaneous nodules on the abdominal wall which were rubbery on palpation. 


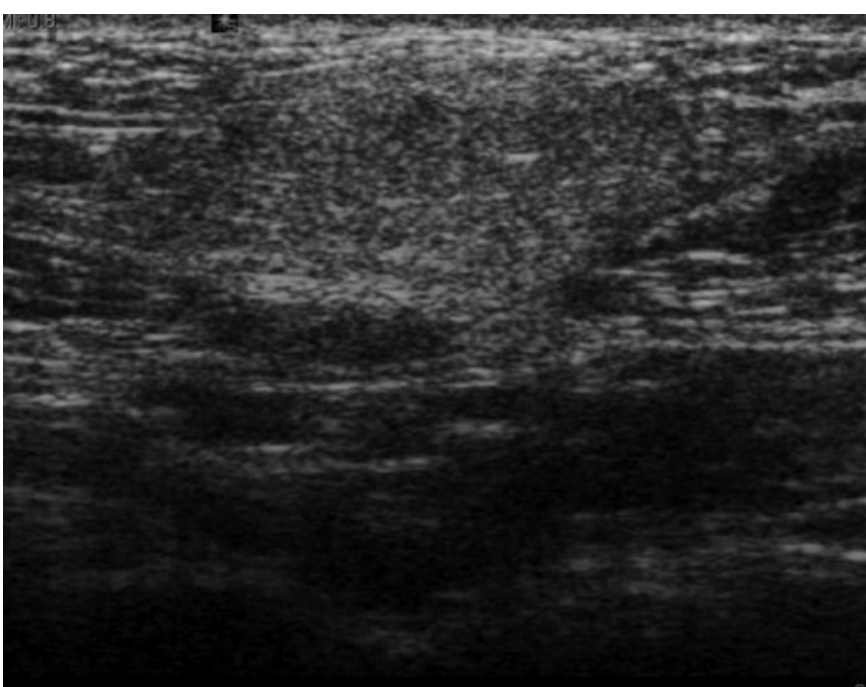

(A)

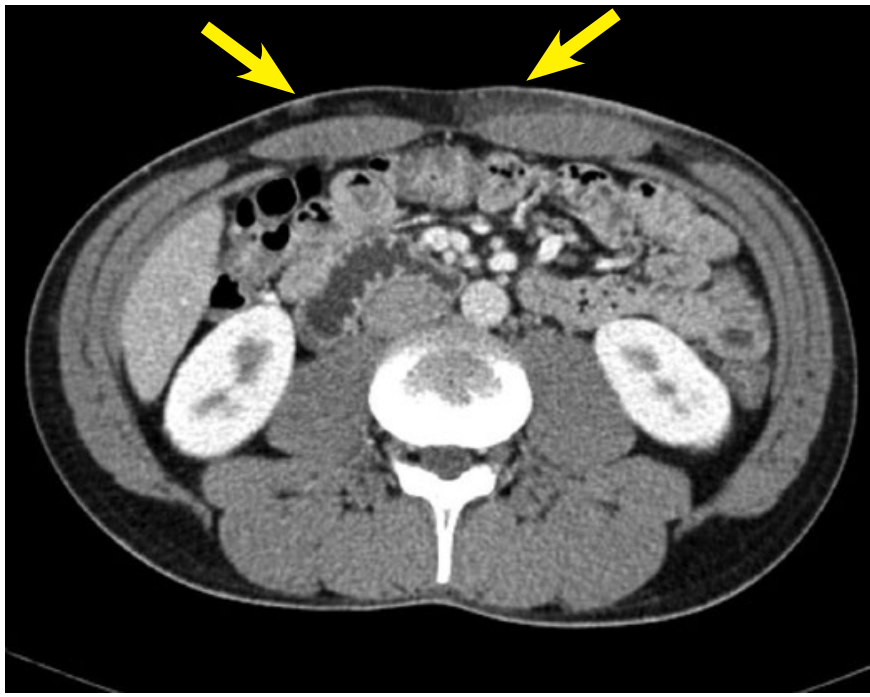

(B)

FIGURE 1. On gray-scale US examination of the lesion in the left abdomen shows ill-defined hyperechoic infiltration (A). Contrast enhanced CT shows multiple mild enhancing nodular and diffuse infiltrative lesions (arrows) in the subcutaneous layer of the anterior abdomen (B).

The nodules were slowly enlarging in size. He was otherwise healthy without weight loss, fever, or chill. On admission, his laboratory tests showed elevated levels of serum lactate dehydrogenase and $\beta 2$-microglobulin.

Ultrasonography was performed on the abdomen. The lesions revealed an ill-defined hyperechoic infiltration (Figure 1A). On abdominal and pelvic CT scanning with contrast enhancement, there were multiple slightly enhancing infiltrative nodular or non-nodular lesions in the subcutaneous layer of the abdominal wall (Figure 1B). One of subcutaneous nodules of the abdominal wall was surgically excised. On histopathologic examina- tion of the excised tissue revealed lymphoid cells diffusely infiltrating through the subcutaneous tissue on hematoxylin \& eosin staining. On immunohistochemical staining, the tumor cells were positive for CD3, CD4 and CD8, but negative for CD56. These histologic findings were consistent with SPTCL.

For systemic surveillance, F-18 FDG PET/CT was performed. Images were obtained 1 hour after an intravenous injection of F-18 FDG (440 MBq) using a PET/CT scanner (Biograph 16 LSO Hi-Res, Siemens, Germany). The patient fasted for 6 hours: the serum glucose level measured before examination was $92 \mathrm{mg} / \mathrm{dl}$. F-18 FDG PET/CT images revealed multiple increased F-18 FDG uptakes corresponding to the infiltrative lesions in the subcutaneous adipose tissue of the chest, back, abdomen and both extremities (Figure 2A-D). However, there was no evidence of lymph node involvement.

The patient received three cycles of CHOP (Cyclophosphamide, Adriamycin, Vincristine and Prednisolone) chemotherapy. After the chemotherapy, a follow-up F-18 FDG PET/CT scan showed a complete metabolic remission of the previous lesions (Figure 3). The patient then received additional three cycles of CHOP (total 6 cycles), and maintained the complete remission with the resolution of all skin lesions. Currently, the patient has been well without recurrence for three years after the last dose of CHOP chemotherapy.

\section{Discussion}

According to the World Health Organization (WHO) classification, lymphoid malignancies are divided largely into T- and B-cell lymphomas, and Hodgkin's disease. T-cell lymphomas are divided into precursor T-cell lymphomas and peripheral T-cell lymphomas. In the category of peripheral T-cell lymphomas, SPTCL has a very low incidence, accounting for less than $1 \%$ of cases of non-Hodgkin's lymphoma. ${ }^{5}$ It was classified as a subtype of cutaneous T-cell lymphoma that preferentially infiltrates the subcutaneous tissue without overt lymph node involvement. ${ }^{2}$

SPTCL occurs in adults as well as in young children, and both sexes are equally affected. Patients present with multiple subcutaneous nodules and plaques, which progress and may ulcerate. ${ }^{6,7}$ Systemic symptoms of this disease are variable, and fever, fatigue, and weight loss may be present. Dissemination to extracutaneous sites is rare. SPTCL may be preceded for years or decades 

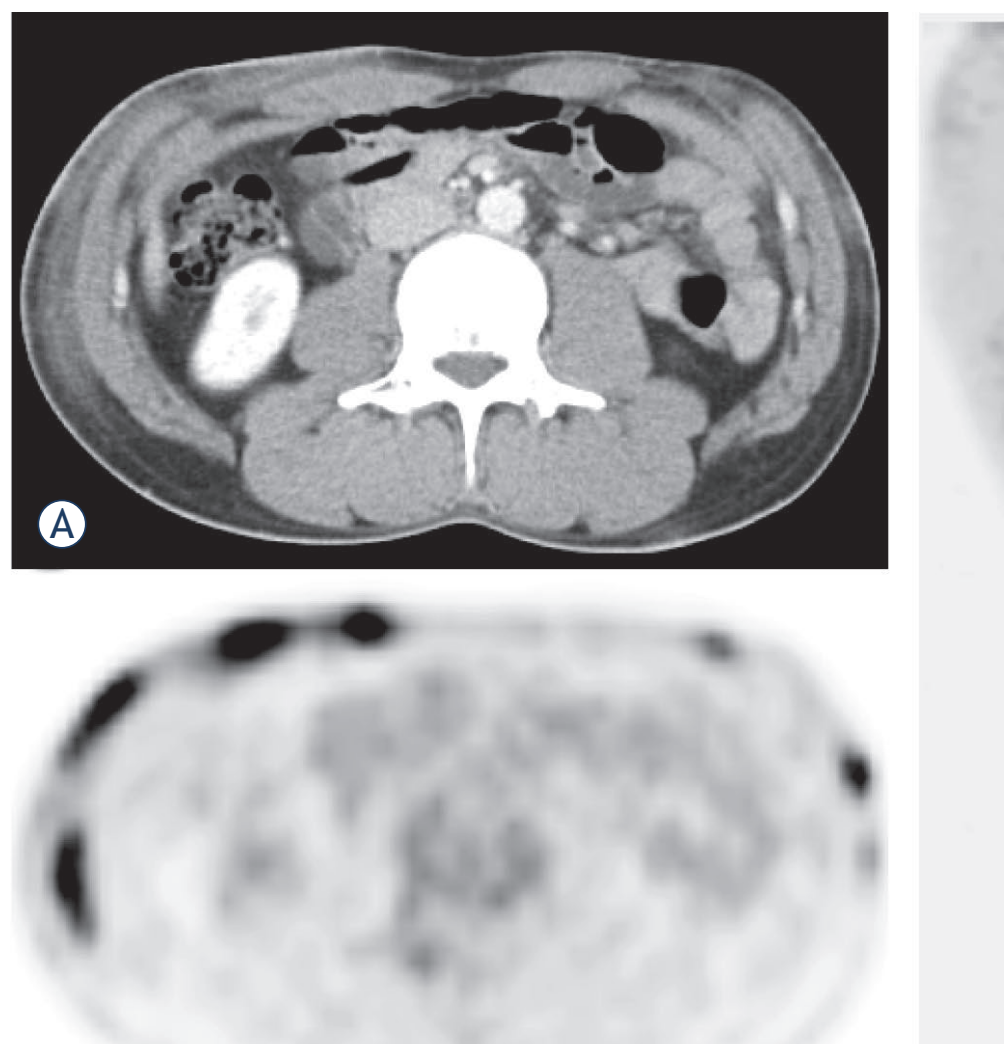

(B)

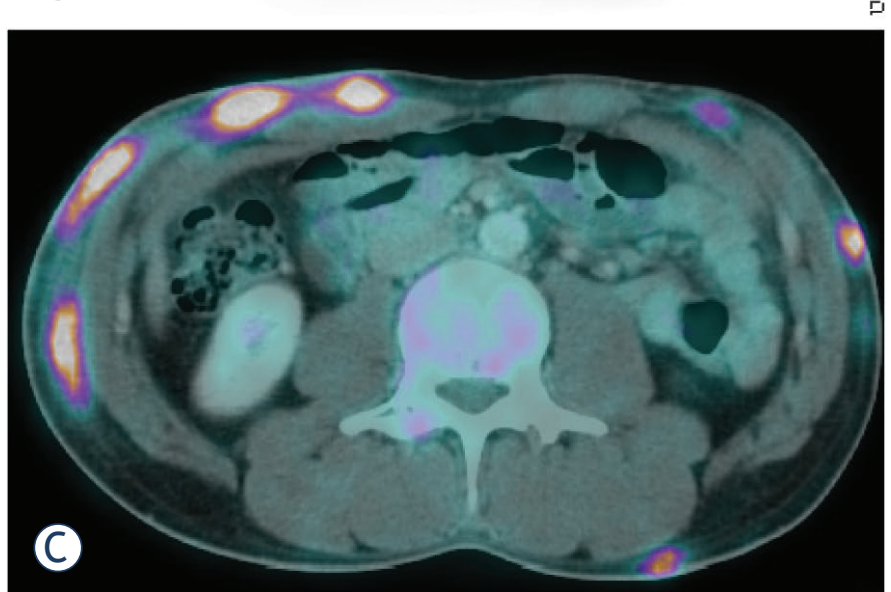

口

(D)

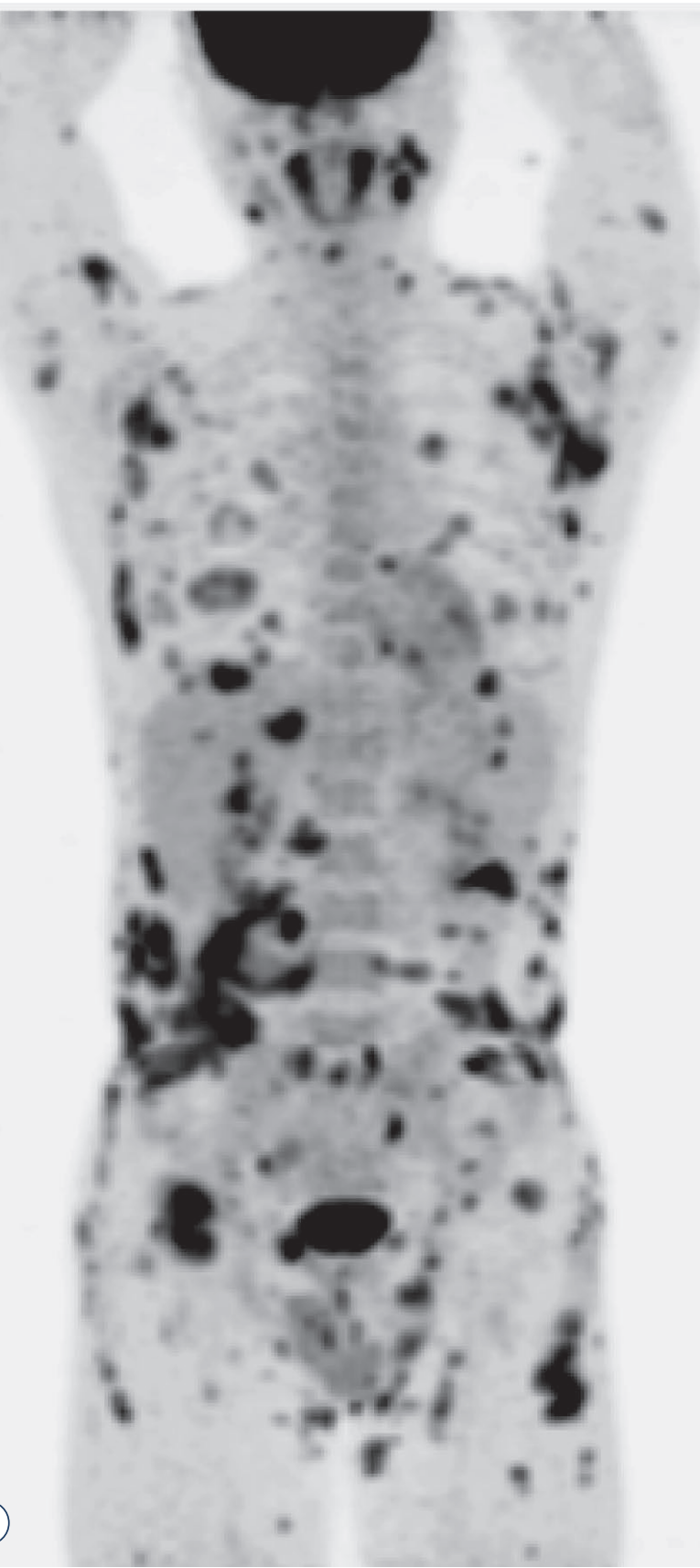

FIGURE 2. Axial contrast-enhanced CT (A), PET (B), and fusion PET/CT (C) images performed for initial staging demonstrated areas of abnormal increased F-18 FDG uptake corresponding to infiltrative changes in the subcutaneous adipose tissue of the anterior abdominal wall. Extensive disease involvement throughout the body with numerous subcutaneous nodules is best visualized on the maximum intensity projection (MIP) image (D).

by a seemingly benign panniculitis. ${ }^{8-10}$ Two clinical courses are reported: Some patients have a protracted course of recurrent spontaneously healing subcutaneous nodules without systemic signs or symptoms; other patients have a rapidly progressive disease and very poor outcome ${ }^{11}$, which is usually due to the development of hemophagocytic syndrome or hepatic dysfunction.
Histologically, SPTCL is characterized by lymphocytic infiltrates confined primarily to the fat lobules in the subcutaneous tissue., 2,12 Rimming of individual fat cells by neoplastic T-cells is a helpful, though not completely specific, diagnostic feature. ${ }^{13}$ In early stage disease, the neoplastic infiltrates may lack significant atypia and a heavy inflammatory infiltrate may predominate..$^{8-10}$ 


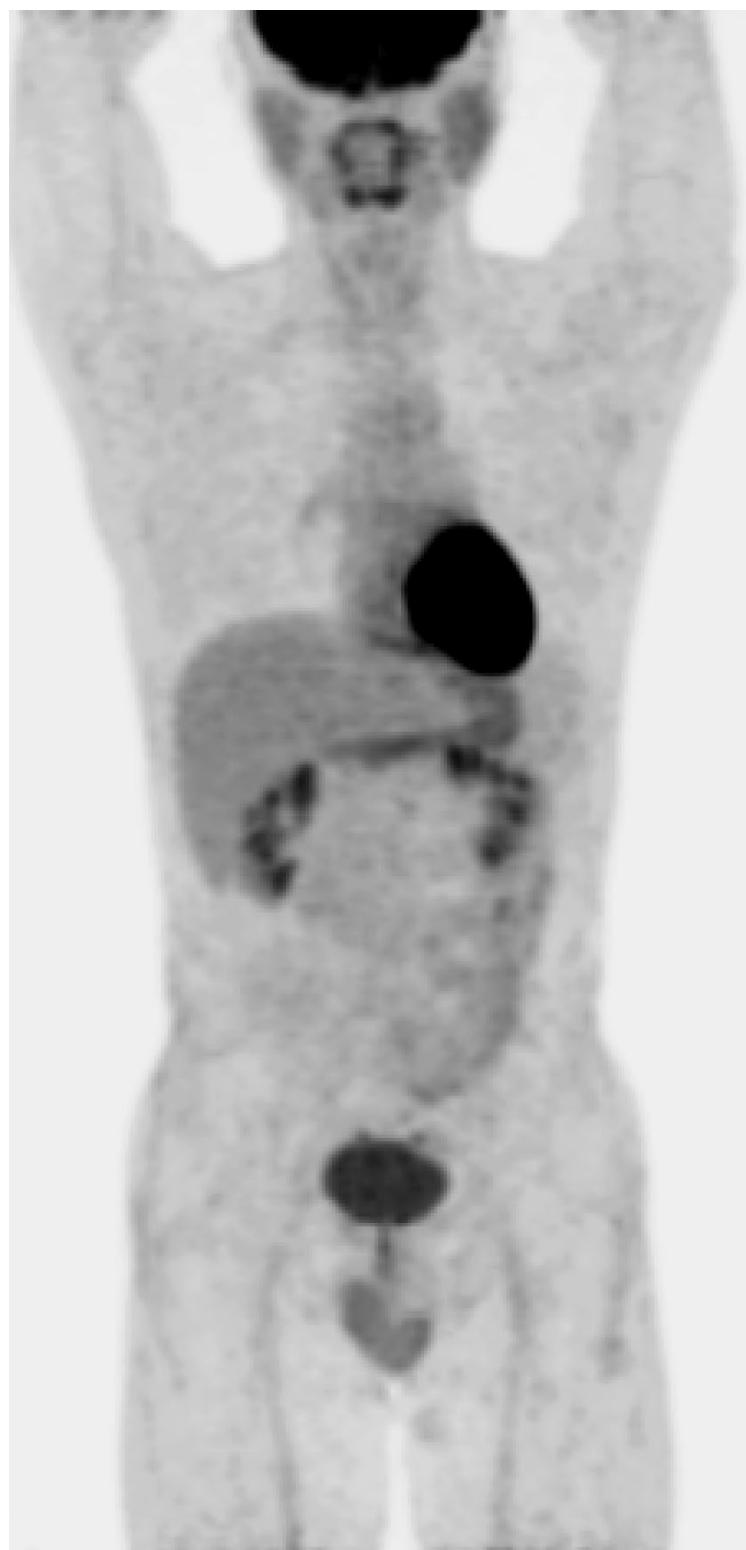

FIGURE 3. After three cycles of chemotherapy, the MIP image of follow-up F-18 FDG $\mathrm{PET} / \mathrm{CT}$ showed complete metabolic remission of the involved lesions.

On CT examination, multiple enhancing nodules are well recognized in the subcutaneous layer of the involved body site. ${ }^{14}$ However, these findings are also noted in inflammatory panniculitis associated with systemic lupus erythematosus or rheumatoid arthritis, subcutaneous metastases from malignant melanoma or breast cancer, and nodules originating from bacterial and fungal infections or from parasitic infestations. ${ }^{15}$

The F-18 FDG PET/CT has emerged as the standard of care for staging, monitoring of the response to therapy, and the detection of disease re- currence in the majority of oncological patients as well as in patients with Hodgkin's and aggressive non-Hodgkin's lymphomas. ${ }^{16-18}$ However, limited studies have been published in the literature on the value of F-18 FDG PET or PET/CT for the evaluation of cutaneous lymphoma. Moreover, only a few cases of SPTCL have been reported describing the findings of F-18 FDG PET or PET/CT. Most studies demonstrated that the subcutaneous lesions of SPTCL showed an FDG-avidity and indicated the superiority of PET/CT over CT alone in detecting nodal involvement. ${ }^{19-22}$ In our case of SPTCL, F-18 FDG PET/CT detected many lesions with greater sensitivity than did physical examination or CT. These studies reported that F-18 FDG PET was valuable in monitoring the treatment response and detecting the extracutaneous lesion in SPTLC. ${ }^{19,23}$

Although further evaluations are needed, the findings in our case suggest that F-18 FDG-PET/CT is a valuable tool for diagnostic work-up, staging and response monitoring in patients with SPTCL as well as those with other FDG-avid lymphoma.

\section{References}

1. Willemze R, Jaffe ES, Burg G, Cerroni L, Berti E, Swerdlow SH, et al. WHOEORTC classification for cutaneous lymphomas. Blood 2005; 105: 3768-85.

2. Gonzalez CL, Medeiros $\amalg$, Braziel RM, Jaffe ES. T-cell lymphoma involving subcutaneous tissue: a clinicopathologic entity commonly associated with hemophagocytic syndrome. Am J Surg Pathol 1991; 15: 17-27.

3. Horvat M, Jezersek Novakovic B. Effect of response quality and line of treatment with rituximab on overall and disease-free survival of patients with B-cell lymphoma. Radiol Oncol 2010; 44: 232-8.

4. Salhany KE, Macon WR, Choi JK, Elenitsas R, Lessin SR, Felgar RE, et al. Subcutaneous panniculitis-like T-cell lymphoma: clinicopathologic, immunophenotypic, and genotypic analysis of alpha/beta and gamma/delta subtypes. Am J Surg Pathol 1998; 22: 881-93.

5. Paulli M, Berti E. Cutaneous T-cell lymphomas (including rare subtypes): current concepts II. Haematologica 2004; 89:1371-2.

6. Willemze R, Jansen PM, Cerroni L, Berti E, Santucci M, Assaf C, et al. Subcutaneous panniculitis-like T-cell lymphoma: definition, classification, and prognostic factors: an EORTC Cutaneous Lymphoma Group Study of 83 cases. Blood 2008; 111: 838-45.

7. Kasper DL, Braunwald E, Fauci AS, Hauser SL, Longo DL, Jameson JL Harrison's principles of internal medicine. New York: McGraw-Hill; 2005. p. 654 .

8. Weenig RH, Ng CS, Perniciaro C. Subcutaneous panniculitis-like T-cell lymphoma. Am J Dermatopathol 2001; 23: 206-15.

9. Hoque SR, Child FJ, Whittaker SJ, Ferreira S, Orchard G, Jenner K, et al Subcutaneous panniculitis-like T-cell lymphoma: a clinicopathological, immunophenotypic and molecular analysis of six patients. Br J Dermatol 2003; 148: $516-25$.

10. Marzano AV, Berti E, Paulli M, Caputo R. Cytophagocytic histiocytic panniculitis and subcutaneous panniculitis-like T-cell lymphoma. Arch Dermatol 2000; 136: 889-96.

11. Perniciaro C, Zalla MJ, White JW Jr, Menke DM. Subcutaneous T-cell lymphoma. Report of two additional cases and further observations. Arch Dermatol. 1993, 129: 1171-6. 
12. Takeshita M, Imayama S, Oshiro Y, Kurihara K, Okamoto S, Matsuki Y, et al. Clinicopathologic analysis of 22 cases of subcutaneous panniculitis-like CD56- or CD56+ lymphoma and review of 44 other reported cases. Am J Clin Pathol 2004; 121: 408-16.

13. Massone C, Chott A, Metze D, Kerl K, Citarella L, Vale E, et al. Subcutaneous, blastic natural killer (NK), NK/T-cell and other cytotoxic lymphomas of the skin: a morphologic, immunophenotypic and molecular study of 50 patients. Am J Surg Pathol 2004; 28: 719-35.

14. Lee HJ, Im JG, Goo JM, Kim KW, Choi BI, Chang KH, et al. Peripheral T-Cell lymphoma: spectrum of imaging findings with clinical and pathologic features. RadioGraphics 2003; 23: 7-26.

15. Kang BS, Choi SH, Cha HJ, Jung YK, Lee JH, Jeong AK, et al. Subcutaneous panniculitis-like T-cell lymphoma: US and CT findings in three patients. Skeletal Radiol 2007; 36: S67-71.

16. Hodolic M. Role of $18 \mathrm{~F}$-choline PET/CT in evaluation of patients with prostate carcinoma. Radiol Oncol 2011; 45: 17-21.

17. Huić $D$, Mutvar A, Kinda-Bašić $S$, Aurer T, Ciglar1M, Grošev D, et al. Negative predictive value of F-18-FDG coincidence PET in patients with Hodgkin's disease and a residual mass after therapy: a retrospective diagnostic test study. Radiol Oncol 2009; 43: 258-63.

18. Jerusalem G, Hustinx R, Beguin Y, Fillet G. Evaluation of therapy for lymphoma. Semin Nucl Med 2005; 35: 186-96.

19. Ravizzini G, Meirelles GS, Horwitz SM, Grewal RK. F-18 FDG uptake in subcutaneous panniculitis-like T-cell lymphoma. Clin Nucl Med 2008; 33: 903-5.

20. Kong EJ, Cho IH, Chun KA, Bae YK, Chol JH, Hyun MS. F-18 FDG PET/CT findings of subcutaneous panniculitis-like T-cell lymphoma: a case report. NuCl Med Mol Imaging 2009; 43: 240-4.

21. Tsai EY, Taur A, Espinosa L, Quon A, Johnson D, Dick S, et al. Staging ac curacy in mycosis fungoides and sezary syndrome using integrated positron emission tomography and computed tomography. Arch Dermatol 2006; 142: $577-84$.

22. Kuo PH, McClennan BL, Carlson K, Wilson LD, Edelson RL, Heald PW, et al. FDG-PET/CT in the evaluation of cutaneous T-cell lymphoma. Mol Imaging Biol 2008; 10: 74-81.

23. Rodriguez VR, Joshi A, Peng F, Rabah RM, Stockmann PT, Savaşan S. Positron emission tomography in subcutaneous panniculitis-like T-cell lymphoma. Pediatr Blood Cancer 2009; 52: 406-8. 